\title{
Development of a mobile application for manual traffic counts
}

\author{
Mohammad Ghanim ${ }^{1, *}$ and Khalid Khawaja ${ }^{2}$ \\ ${ }^{1}$ Department of Civil and Architectural Engineering, Qatar University, Doha, Qatar \\ ${ }^{2}$ Office of Academic Affairs, RIT Dubai, Dubai, UAE
}

\begin{abstract}
The use of technology in performing traffic counts has become widely used by many transportation agencies and private sectors. Different technologies have been employed to perform such counts, such as inductive loops, image processing, Bluetooth technologies, and laser and infrared technologies. However, the use of these advanced technologies come with extra cost to develop, install, and maintain. While the use of advanced technologies provide reliable traffic counts and vehicle classifications data, the use of manual traffic is an inevitable task. Manual traffic counts can serve different purposes, such as performing quality control or conducting short-term traffic survey. Manual traffic counts are usually conducted with the use of the traditional paper-and-pencil approach or the use of hand-held devices that are specifically developed to assess performing manual traffic counts. In this paper, the development of a mobile app that can be used to assist those who need to perform manual traffic counts. The app can be used by many public agencies, private sectors, and college students to collect their traffic data. It also provides users with the capabilities of sharing their traffic counts instantly, where traffic counts can be extracted and processed quickly by other parties.
\end{abstract}

\section{Introduction}

The advances of technology nowadays allow transportation and engineers to collect traffic counts using many different technologies that vary in their complexity, type of collected traffic data, and price, and mobility. However, there is always a need to perform manual traffic counts for different reasons, such as the associated costs and quality control needs. There are many portable products and hand-held devices that are used to facilitate manual traffic counts. Usually these devices are bundled with a special software to abstract, process, and summarize traffic counts.

The use of mobile apps nowadays has spread widely and rapidly over the last 10 years. There are over three million mobile apps that are developed and operated under the leading app stores, for different smartphone operation system platforms (i.e., iOS, Android, Amazon, Windows Phone, and BlackBerry) [1-4]. Furthermore, the hardware components themselves are developed with integrated devices that will increase their usability, such as

\footnotetext{
* Corresponding author: mghanim@qu.edu.qa
} 
global positioning systems (GPS), front and rear high resolution cameras, and accelerometers.

The use of smart phone and mobile applications have gained popularity over the last decade, as can be seen in these examples [5-8].

Despite the exponential growth of using mobile apps to do many different tasks, its usability to perform manual traffic counts are very limited. Although this can be partially associated with the fact that there is no comprehensive mobile app that can do several traffic counts functionalities.

This paper illustrates the development of a mobile app that is comprehensive enough to assist performing most of the manual traffic count needs, such as mainstream flow counts, pedestrians' counts, turning movement counts, and vehicle classification.

\section{Mobile app development motivation}

Although there are several hand-held devices that are used to collect manual traffic data, most of the portable traffic counters are usually expensive, when compared to widely spread tablets and smartphones. Traffic counts data cannot be easily transferred for further processing. Their size is big when compared to the area of hand palms. Moreover, they have limited functionalities that does not exceed recording traffic counts. Therefore, the need for a manual traffic count system that is price-insensitive, highly portable, with on-demand availability is needed.

\section{Traffic abacus functionalities}

In this project, a mobile app called "Traffic Abacus" that works under Android's OS is being developed [9]. The Android OS is used due to its wide usage. The developed app is designed to allow several manual traffic counts needs, covering most of the scenarios where manual traffic counts are suited. These functionalities are:

- 4-leg Intersections: w/o U-Turns and w/o pedestrians.

- 4-leg Intersections: w/o U-Turns and with pedestrians.

- 4-leg Intersections/Roundabouts: with U-Turns and w/o pedestrians.

- 4-leg Intersections/Roundabouts: with U-Turns and with pedestrians.

- Vehicle Classification: according to the FHWA Scheme "F".

- Directional Mainstream Traffic Counts: can be classified per lane.

Although roundabouts are not specifically listed as an option, a four-leg intersection with U-Turns. As can be seen in Fig. 1, a snapshot is taken for each of the previous functionalities. 

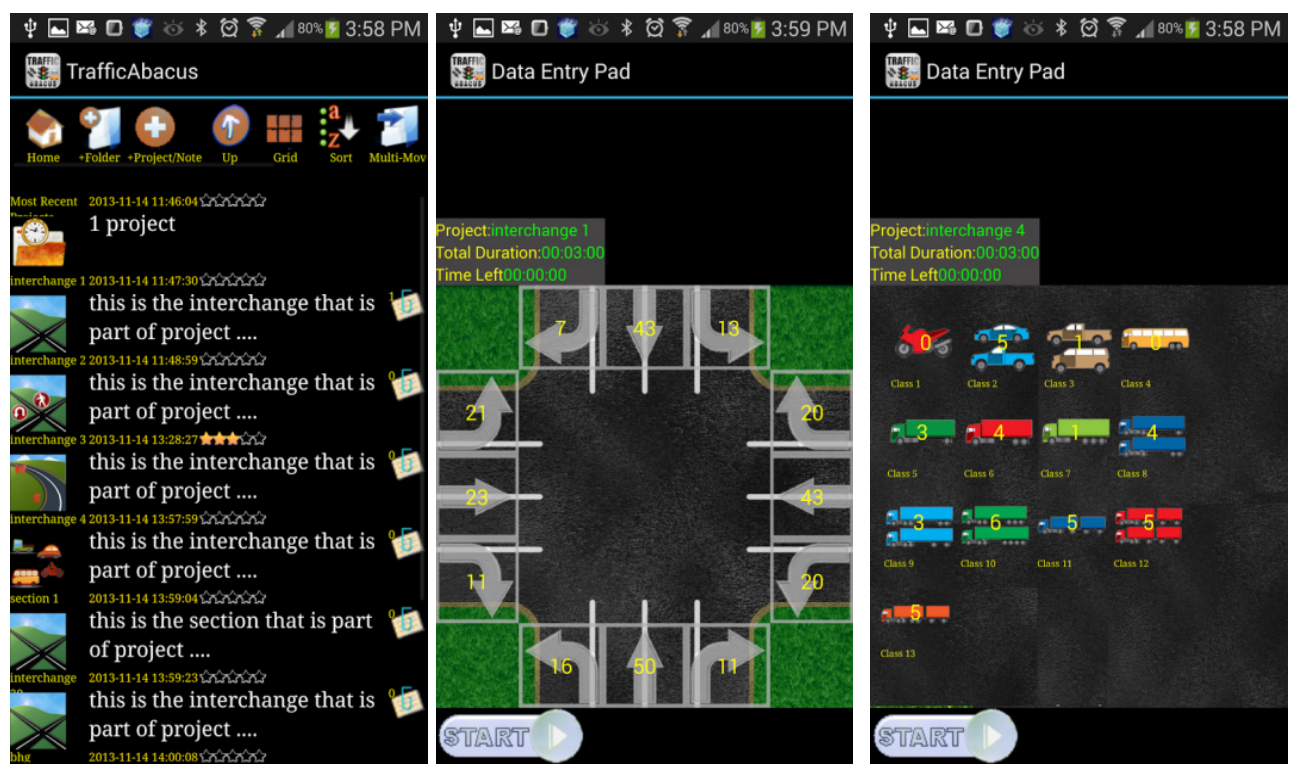

Fig. 1: Traffic Abacus Application Interface - Sample Snapshots.

\section{Traffic abacus architecture}

This application is developed to work on Android devices. The following broad objectives were stated for the application project:

- The application must support all the basic traffic count scenarios:

- The application must function properly on a single device without internet connection

- The application must support ability to link notes and media files (audio, photo and video) to each counting session

- The application must support ability to group various sessions together

- The application must allow sharing of collected data

As a result, all the scenarios that were presented in the previous section were implemented in a standalone application with the architecture shown in Fig. 2 and Fig. 3. The user can initiate a project for a scenario data collection and group projects in a folder. The user can also attach notes and media files to any project. A local database was used to store collected data and any associated notes. The database allows for the operation of the application independent from any network connection. An export operation leads to the generation of an XML file with all the data. The XML file is able to represent a single project, a folder that contains several projects, or even several folders. This XML file can then be sent to any user of the Traffic Abacus application who can import the file to get the same view. This allows and promote team collaboration and simultaneous team data collection. In addition, this architecture lends itself to future enhancement that allows server based real-time synchronization of team data utilizing the XML file as the data exchange medium. The next section gets into some more details related the add-ons gained from data collection on the mobile. 


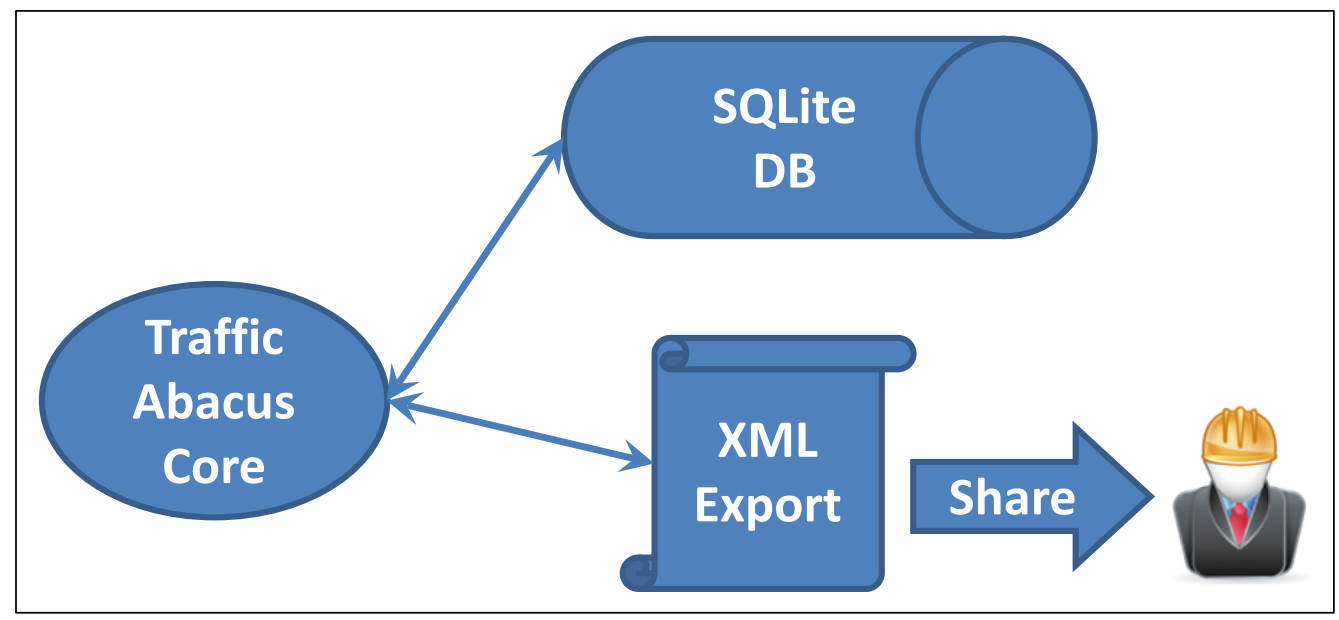

Fig. 2: Application Architecture.

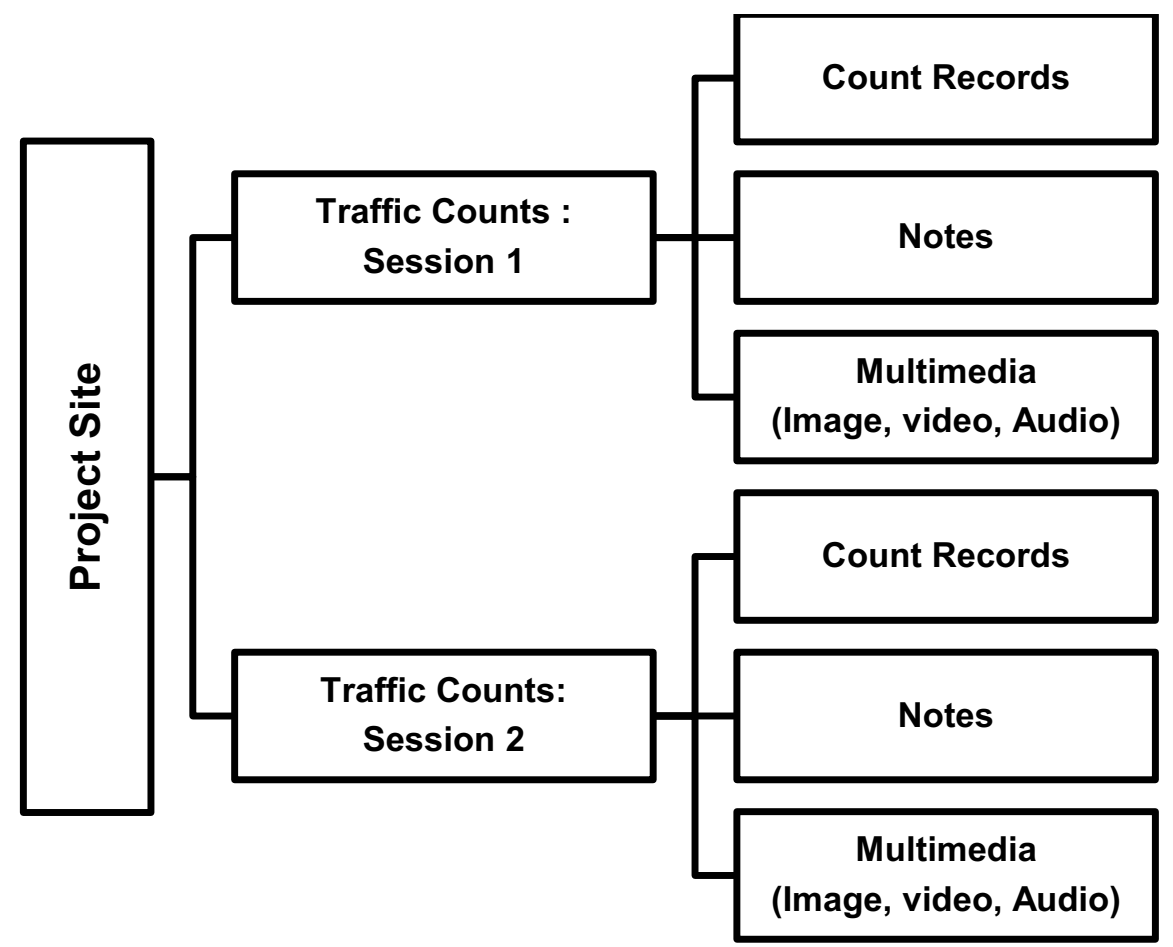

Fig. 3: Hierarchical Presentation of a Project Site Contents.

\section{Traffic abacus application add-ons}

In order to take full advantage of technologies available in mobile apps, the following features are also implemented: 


\subsection{Multiple sessions records:}

Once a project is initiated, the app allows the user to record different count sessions, such as morning, mid-day, and evening peak periods. Therefore, the count sessions can be deleted or processed. They also can be different traffic counts category, such intersection counts and vehicle classifications.

\subsection{Traffic counts timestamp:}

A timestamp is created for each click. This timestamp is stored in the local database. Each timestamp contains the time, movement type, and project ID. This data can be viewed in the application or, as explained earlier, exported in an XML file that can be viewed using any web browser or XML viewer. Furthermore, traffic counts can be processed using any available software, such as Excel and Google Sheets. One of the advantages of using the timestamp is that data can be processed and recorded at a user-defined interval. For instance, it can be aggregated every five-minute, 15-minute or one hour intervals, as can be seen in Fig. 4.

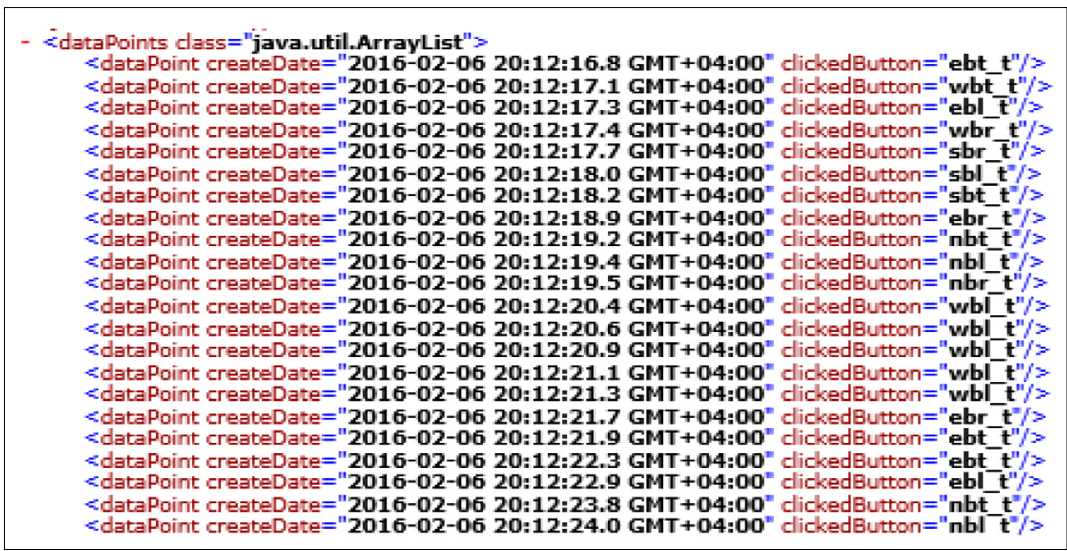

Fig. 4: Data points presentation in the Traffic Abacus XML file.

\subsection{Online sharing:}

Once the traffic count session is over and the XML file is created and saved on the smartphone or tablet, it can be instantly shared with anyone. Therefore, processing and extracting the data can be done almost immediately.

\subsection{Note attachment:}

This functionality allows the traffic surveyor to attach notes associated with a particular count session. These notes can be anything related to the project, such as incident occurrences and weather condition. These notes can be a valuable reference if proper notes are taken, and an unexpected traffic trend is observed based on data records. 


\subsection{Multimedia records:}

In addition to typed notes, the app allows the user to take images, audio videos records and attach them to a particular project. These types of records are important to identify several site features, such as geometric layout. Voice and video records are also helpful and they can serve many other purposes.

Fig. 5 shows the panel that allows addition of projects as well as addition of attached data. While notes and media files can be selected to include with any folder including any number of projects (second panel in the figure), they are the only objects that can be selected when we are adding material as attachments to a particular project (first panel in the figure). Figure 5 provides a hierarchical presentation of attached data to any project.

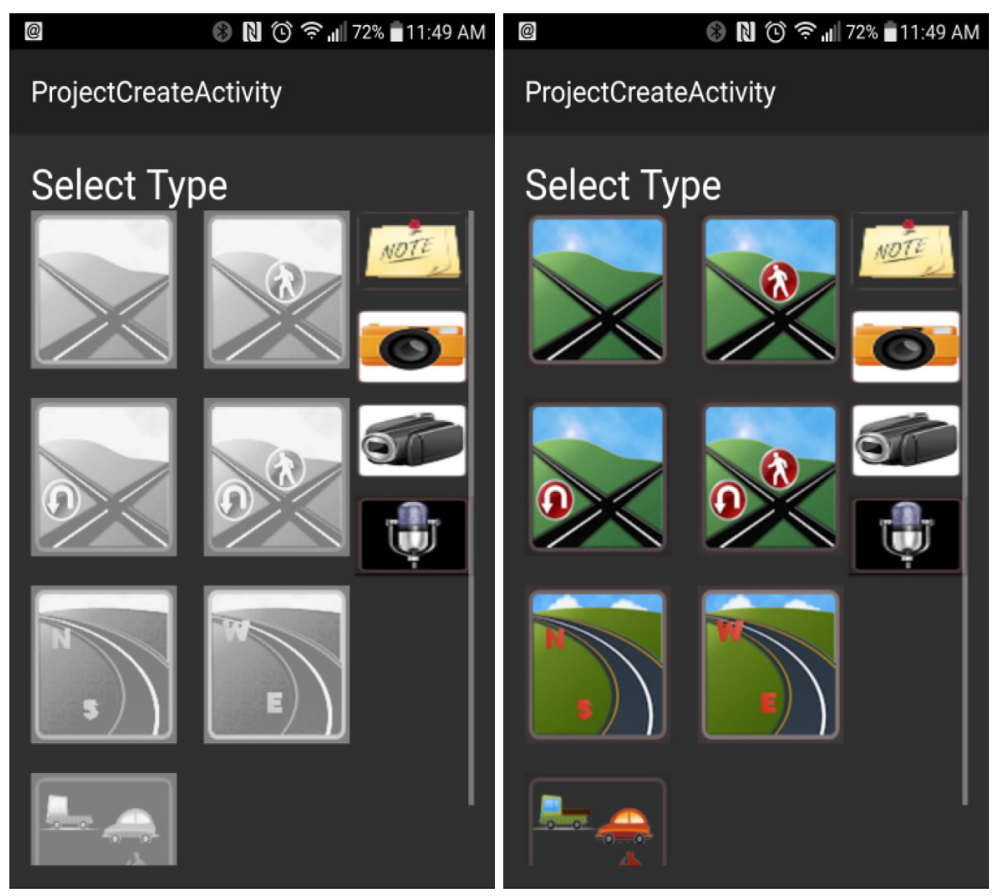

Fig. 5: Panel that allows for the attachment of various data types.

\section{Students' feedback}

This mobile app has been used by students from two different universities in the Middle East, namely the American University in Dubai and Qatar University. The students chose to use this application due to the unavailability of professional traffic counters developed by the industry, or the lack of proper training on these counters and extract count data. Some of the students chose to use this application because of the ease of use. The students used the app to collect traffic counts for their classroom and research projects.

A special survey is currently being developed to collect student's feedback on their experience of using such a mobile application to collect traffic data manually. This survey will assess the usefulness and the convenience of collecting traffic data. It will also be used overcome any shortage that the students might have experienced. Based on the survey results, more enhancement can be developed to assure full functionality for the mobile application. 


\section{Conclusion}

In this project, the development of a mobile app called "Traffic Abacus" that works under Android's operating system has been described. This app is designed to provide a practical tool for traffic engineering students and professional personnel's to collect manual traffic counts. During the development phase of this app, the different needs of manual traffic counts and data collections were considered. This includes the need for counting turning movements at intersections, counting pedestrians' movements, stream traffic counts, and vehicle classification studies.

This paper demonstrates the fundamental assistance that the mobile application can offer to traffic engineering students and professionals when manual traffic counts are needed. Additionally, it provides additional add-ons that are important to facilitate the process of collecting traffic data. Some of these features are the ability to add multiple count sessions under one project, the ability to attach images, videos, and notes. Furthermore, this app provides the ability share and export project immediately once the count session is over.

\section{Recommendations}

While this paper demonstrates the functionalities of first phase in this project, further developments will follow. Some of the future plans is to add the Global Positioning System information to the traffic count sessions, through the XML file. Furthermore, the research team is evaluating the potential of expanding the platform of this project to include other operation systems, such as iOS and Windows Phone OS.

\section{References}

1. Allan, A., Learning iOS Programming: From Xcode to App Store. 2013: " O'Reilly Media, Inc.".

2. Nurmi, D., et al. The eucalyptus open-source cloud-computing system. in Cluster Computing and the Grid, 2009. CCGRID'09. 9th IEEE/ACM International Symposium on. 2009. IEEE.

3. Allen, S., V. Graupera, and L. Lundrigan, Pro smartphone cross-platform development: iPhone, blackberry, windows mobile and android development and distribution. 2010: Apress.

4. Yan, T., et al. Fast app launching for mobile devices using predictive user context. in Proceedings of the 10th international conference on Mobile systems, applications, and services. 2012. ACM.

5. Shi, C., et al. Routine driving infotainment app: Gamification of performance driving. in Adjunct Proceedings of the 4th International Conference on Automotive User Interfaces and Interactive Vehicular Applications. 2012.

6. Wang, T., et al. WalkSafe: a pedestrian safety app for mobile phone users who walk and talk while crossing roads. in Proceedings of the Twelfth Workshop on Mobile Computing Systems \& Applications. 2012. ACM.

7. Ozbay, K., et al., Big data and calibration and validation of traffic simulation models. Traffic and Transportation Simulation: Looking Back and Looking Ahead: Celebrating, 2014. 50: p. 92-122.

8. Gavin, M., et al. A cycle route planner mobile-app for Dublin city. in Irish Transportation Research Network Conference (ITRN2011), 31 Aug-1 Sep 2011, University College Cork, Cork, Ireland. 2011. Irish Transportation Research Network.

9. Rogers, R., et al., Android application development: Programming with the Google SDK. 2009: O'Reilly Media, Inc. 